\title{
Analysis of p53 mutations for a mutational signature in human intrahepatic cholangiocarcinoma
}

\author{
SHAHID A. KHAN ${ }^{1}$, SIMON D. TAYLOR-ROBINSON ${ }^{1}$, PAUL L. CARMICHAEL ${ }^{2}$, \\ NAGY HABIB $^{3}$, NICHOLAS R. LEMOINE ${ }^{4}$ and HOWARD C. THOMAS ${ }^{1}$ \\ ${ }^{1}$ Liver Unit, Department of Medicine A, St Mary's Campus; ${ }^{2}$ Genetic Toxicology, SEAC - Safety and Environmental \\ Assurance Centre, Unilever Colworth, Sharnbrook, Bedford; ${ }^{3}$ Department of Surgery, and ${ }^{4}$ Cancer Research UK \\ Molecular Oncology Unit, Hammersmith Campus, Faculty of Medicine, Imperial College London, UK
}

Received September 22, 2005; Accepted November 11, 2005

\begin{abstract}
Cholangiocarcinoma development may be related to cholangiocyte DNA damage from genotoxic compounds in bile. We have previously shown that human biliary tissue is exposed to genotoxic agents, as evidenced by the presence of DNA adducts. Establishing the presence of a 'mutational signature' in tumour suppressor genes from tumour tissue provides a means of linking cause and effect in human cancer. Inactivation of p53, known to have 'hot-spots' for particular chemical carcinogens, has previously been linked to human cholangiocarcinoma. However, previous p53 studies have focused on exons 5-8, potentially missing gene alterations at other sites. This study examined the putative link between environmental carcinogens and intrahepatic cholangiocarcinoma by analysing DNA from 31 patients for complete p53 mutational signatures, using single strand conformational polymorphism and polymerase chain reaction. All mutations found were compared to known p53 mutations in cholangiocarcinoma and to mutations induced by environmental mutagens, as described in p53 databases. Five non-silent p53 mutations were found, including three new frameshift mutations and two new intron mutations which have not previously been reported in cholangiocarcinoma. Two frameshifts were due to deletions and the third due to an insertion in exon 5. There was no predominant mutational spectrum amongst the set of cholangiocarcinoma samples
\end{abstract}

Correspondence to: Dr S.A. Khan, Liver Unit, Department of Medicine A, 10th Floor, QEQM Wing, Faculty of Medicine, Imperial College London, St Mary's Hospital, South Wharf Street, London W2 1NY, UK

E-mail: shahid.khan@imperial.ac.uk

Abbreviations: HCC, hepatocellular carcinoma; AFB1, aflatoxin B1; IARC, International Agency for Research on Cancer; SSCP, single strand conformational polymorphism; PCR, polymerase chain reaction

Key words: chemical carcinogens, genotoxins, IARC database studied, or on combining these mutations with the dataset of known p53 mutations in cholangiocarcinoma. Several reasons may explain this, including lack of data outside exons 5-8, bias in mutation reporting, the involvement of mutations in non-coding regions or genes other than $\mathrm{p} 53$, or the possibility that there is no carcinogenic specific agent and therefore no signature.

\section{Introduction}

Mortality from cholangiocarcinoma is increasing in the USA and other industrialised countries (1). The cause is unknown. We postulate that the rise in cholangiocarcinoma mortality may represent, at least in part, an environmental change and specifically, increased exposure to environmental carcinogens.

The covalent binding of genotoxic carcinogens to DNA and the resulting mutations are not random, but rather show base and positional specificity, leading to high-frequency mutations in specific short sequences of DNA in a target gene. The characterisation of such sequence-specific DNA lesions along a target gene in human cancer can be correlated with known mutational spectra induced by environmental carcinogens. Establishing the presence of the same signature in oncogenes or tumour suppressor genes in tumour tissue from individuals exposed to such carcinogens can provide a powerful means of linking cause and effect in human cancer $(2,3)$.

Mutations of the tumour suppressor gene p53 have previously been attributed as the link between chemical carcinogens and certain human malignancies. For example, hepatocellular carcinoma (HCC) in some areas of the world, such as China and parts of sub-Saharan Africa, has been strongly associated with dietary exposure to AFB1. In these areas, one dominant mutational hot-spot is found in up to 50\% of HCCs: a GC to TA transversion at the third position of codon 249 of the p53 gene, which renders p53 inactive (4). This mutation is induced by chemically reactive forms of AFB1, and is uncommon (1\%) in HCC samples from Japan and Western countries, where aflatoxin exposure is low (4).

p53 abnormality has been implicated in cholangiocarcinoma by immunohistochemical $(5,6)$ and molecular epidemiological studies, including gene sequencing studies, mainly of exons 
Table I. A, Primers used for p53 exons.

\begin{tabular}{ll}
\hline P53 exon 1 for & TGTAAAACGACGGCCAGTTCCCATGTGCTCAAGACTGG \\
P53 exon 1 rev & CAGGAAACAGCTATGACCGCCCGTGACTCAGAGAGGAC \\
P53 exon 2 for & TGTAAAACGACGGCCAGTTGGAAGTGTCTCATGCTGGATC \\
P53 exon 2 rev & CAGGAAACAGCTATGACCAATTTCGCTTCCCACAGGTC \\
P53 exon 3 for & TGTAAAACGACGGCCAGTAATTCATGGGACTGACTTTCTGC \\
P53 exon 3 rev & CAGGAAACAGCTATGACCTGGGTCTTCAGTGAACCATTGTT \\
P53 exon 4 for & TGTAAAACGACGGCCAGTCCTGGTCCTCTGACTGCTCTTT \\
P53 exon 4 rev & CAGGAAACAGCTATGACCGAAGTCTCATGGAAGCCAGCC \\
P53 exon 5 for & TGTAAAACGACGGCCAGTTCACTTGTGCCCTGACTTTCAA \\
P53 exon 5 rev & CAGGAAACAGCTATGACCCAGTGAGGAATCAGAGGCCTG \\
P53 exon 6 for & TGTAAAACGACGGCCAGTGGTCCCCAGGCCTCTGATT \\
P53 exon 6 rev & CAGGAAACAGCTATGACCGACAACCACCCTTAACCCCTC \\
P53 exon 7 for & TGTAAAACGACGGCCAGTTTGCCACAGGTCTCCCCA \\
P53 exon 7 rev & CAGGAAACAGCTATGACCGGGCACAGCAGGCCAG \\
P53 exon 8 for & TGTAAAACGACGGCCAGTGATTTCCTTACTGCCTCTTGCTTCT \\
P53 exon 8 rev & CAGGAAACAGCTATGACCAGTGAATCTGAGGCATAACTGCAC \\
P53 exon 9 for & TGTAAAACGACGGCCAGTTGCAGTTATGCCTCAGATTCACTT \\
P53 exon 9 rev & CAGGAAACAGCTATGACCTGAGTGTTAGACTGGAAACTTTCCA \\
P53 exon 10 for & TGTAAAACGACGGCCAGTAACTTGAACCATCTTTAACTCAGG \\
P53 exon 10 rev & CAGGAAACAGCTATGACCCCAACCTAGGAAGGCAGGG \\
P53 exon 11 for & TGTAAAACGACGGCCAGTGCCCTTCAAAGCATTGGTC \\
P53 exon 11 rev & CAGGAAACAGCTATGACCAAATGGAAGTCCTGGGTGC \\
\end{tabular}

B, M13 primers.

5-8. Collectively, over 90 different p53 mutations have been described in cholangiocarcinoma, including the IARC specialised p53 database, which contains information on all reported p53 mutations (7). Given the marked geographical variation cholangiocarcinoma shows in its incidence and mutation rates, a review concluded that the presence and type of p53 mutation in cholangiocarcinoma is dependent on environmental factors, including differences in the nature or dose of exposure to environmental carcinogens, which vary in different populations (8).

We hypothesise that intrahepatic cholangiocarcinoma is caused by exposure to an environmental mutagen, and that this may be reflected in a consistent mutational spectrum of the p53 gene. Previous p53 mutation studies have focused on exons 5-8 only, potentially missing mutations that could be relevant in terms of a functional role and/or a mutational signature. Our aim was to examine the putative link between environmental carcinogens and human cholangiocarcinoma, by analysing DNA from human cholangiocarcinoma samples for complete p53 mutational signatures by analysis of the entire cDNA gene sequence (all 11 exons), and comparing any mutations found to known p53 mutational spectra induced by environmental mutagens, which have been described in the IARC p53 database.

\section{Materials and methods}

DNA samples from 31 cholangiocarcinoma patients were examined for p53 mutations. The entire p53 cDNA from each sample was initially screened by SSCP, and those exons exhibiting aberrant SSCP bands were fully sequenced by PCR. Each sequenced sample was amplified by PCR twice: the first time was using fluorescently labelled primers, and the PCR products were analysed by SSCP. Any abnormal SSCP traces were amplified from the original template in a second independent reaction using non-labelled primers and sequenced to look for the mutation that caused the SSCP result.

Patients, sample collection and DNA extraction. Tumour tissue samples were collected at surgery from 31 intrahepatic cholangiocarcinoma patients (12 male, age range 36-70 years, mean age 56 years) by hepatobiliary surgeons at Hammersmith Hospital, Imperial College London. All patients were resident in the UK. Three out of the 31 patients were of South Asian origin and the remainder were Caucasian. All samples were verified as primary intrahepatic cholangiocarcinomas by histology and radiology, and stored at $-70^{\circ} \mathrm{C}$ until DNA extraction. DNA was extracted from tissue samples by a standard phenol method (9). 
Table II. The 12 different p53 gene alterations found amongst 24 out of the 31 study samples.

\begin{tabular}{|c|c|c|c|}
\hline $\begin{array}{l}\text { Location } \\
- \text { in } / \mathrm{ex} / \mathrm{n} / \mathrm{c}^{\mathrm{a}}\end{array}$ & $\begin{array}{l}\text { Gene alteration } \\
\text { or mutation }\end{array}$ & $\begin{array}{l}\text { Number of patients } \\
\text { (as \% of total) }\end{array}$ & Effect on mRNA \\
\hline 1) in $2 / n 11827$ & $\mathrm{G}>\mathrm{C}$ & $13(42)$ & $\begin{array}{l}\text { Nil. Known polymorphism } \\
\text { of unknown prevalence }\end{array}$ \\
\hline 2) in $3 / \mathrm{n} 11992^{\mathrm{b}}$ & $\mathrm{C}>\mathrm{A}$ & $5(16)$ & Nil \\
\hline 3) in3/n11951 & 16 bp insertion & $6(19)$ & $\begin{array}{l}\text { Nil. Known polymorphism } \\
\text { of unknown prevalence }\end{array}$ \\
\hline 4) ex4/n12139/c72 & $\mathrm{CGC}>\mathrm{CCC}$ & $11(35)$ & $\begin{array}{l}\mathrm{ARG}>\mathrm{PRO} \\
\text { Known polymorphism with } \\
\text { wide-ranging prevalence }\end{array}$ \\
\hline 5) ex $4 / \mathrm{n} 12032 / \mathrm{c} 36$ & $\mathrm{CC} G>\mathrm{CCA}$ & 1 (3) & $\begin{array}{l}\text { PRO>PRO Silent. } \\
\text { Known polymorphism } \\
\text { with } 4 \% \text { prevalence }\end{array}$ \\
\hline 6) ex $4 / \mathrm{n} 12243 / \mathrm{c} 107^{\mathrm{b}}$ & Deletion $\mathrm{T}$ & 1 (3) & $\begin{array}{l}\text { Frameshift: } \\
\text { termination at c180 }\end{array}$ \\
\hline 7) ex5/n13117/c146 & $\mathrm{TG} G>\mathrm{TG} A$ & $2(6)$ & TRP $>$ STOP \\
\hline 8) ex5/n13129-13130/c151 b & Insertion $\mathrm{C}$ & 1 (3) & $\begin{array}{l}\text { Frameshift: } \\
\text { termination at } \mathrm{c} 180\end{array}$ \\
\hline 9) ex5/n13203/c175 & $\mathrm{C} G \mathrm{C}>\mathrm{CAC}$ & $2(6)$ & $\mathrm{ARG}>\mathrm{HIS}$ \\
\hline 10) ex6/n13399/c213 & $\mathrm{CG} A>\mathrm{CG} G$ & $3(10)$ & $\begin{array}{l}\text { ARG }>\text { ARG Silent. } \\
\text { Known polymorphism with } \\
\text { upto } 11 \% \text { prevalence }\end{array}$ \\
\hline 11) ex6/n13332-13333/c191 b & Deletion CT & $2(6)$ & $\begin{array}{l}\text { Frameshift: } \\
\text { termination at c } 207\end{array}$ \\
\hline 12) in $7 / \mathrm{n} 13964^{\mathrm{b}}$ & $\mathrm{G}>\mathrm{C}$ & 1 (3) & Nil \\
\hline
\end{tabular}

${ }^{\mathrm{a}}$ in/ex/n/c, intron or exon/nucleotide/codon (if applicable); ${ }^{\mathrm{b}}$ previously unreported mutations.

Polymerase chain reaction. Primers for p53 were redesigned for all eleven exons with M13 tails using Genbank accession number U94788 as a reference sequence and Primer Design software (Applied Biosystems). Primers were ordered from Interactiva (Ulm, Germany). All primers are detailed in Table I. The primers were designed to enable analysis of exon/intron junctional regions.

SSCP analysis. PCR product $(1 \mu \mathrm{l})$ was mixed with Hi-Di Formamide $(10 \mu \mathrm{l})$ and $0.5 \mu \mathrm{l}$ Genescan ROX-500 size standard (Applied Biosystems, Foster City, USA). The sample was then heat denatured for $2 \mathrm{~min}$ at $95^{\circ} \mathrm{C}$ and snap cooled on ice before loading onto an ABI3100 genetic analyser. Electrophoresis was performed using $5 \%$ native genescan polymer with $10 \%$ glycerol and $1 \mathrm{X}$ TBE injected into a $50 \mu \mathrm{m}$ diameter capillary with length to detector of $36 \mathrm{~cm}$. Consecutive runs were performed at 18,22 and $35^{\circ} \mathrm{C}$ respectively, with sample injection times of $15 \mathrm{sec}$ at $15 \mathrm{kV}$ followed by electrophoresis at $15 \mathrm{kV}$ for $30 \mathrm{~min}$, prior to data collection.
Sequencing. Samples exhibiting band shifts under at least one of the SSCP conditions were subjected to sequence analysis on the ABI3100 using a Big-Dye Terminator Ready Reaction kit (Applied Biosystems). PCRs were initially performed, as detailed above, just using gene specific primers and the sequencing was performed using unlabeled M13 primers. DNA variations were identified in both forward and reverse strands.

Data analysis. Samples were analysed using sequence analysis software and aligned against a known wild-type in the sequence navigator (Applied Biosystems). Traces of both the wild-type and potential mutant were then viewed and mutations identified. Mutations found in the samples were compared to all known p53 mutations in cholangiocarcinoma, as well as to all p53 mutations known to be induced by environmental carcinogens, as contained in the IARC p53 database. The IARC database holds data on all reported p53 mutations arising from various known carcinogens, including aflatoxins, aromatic amines, 
A

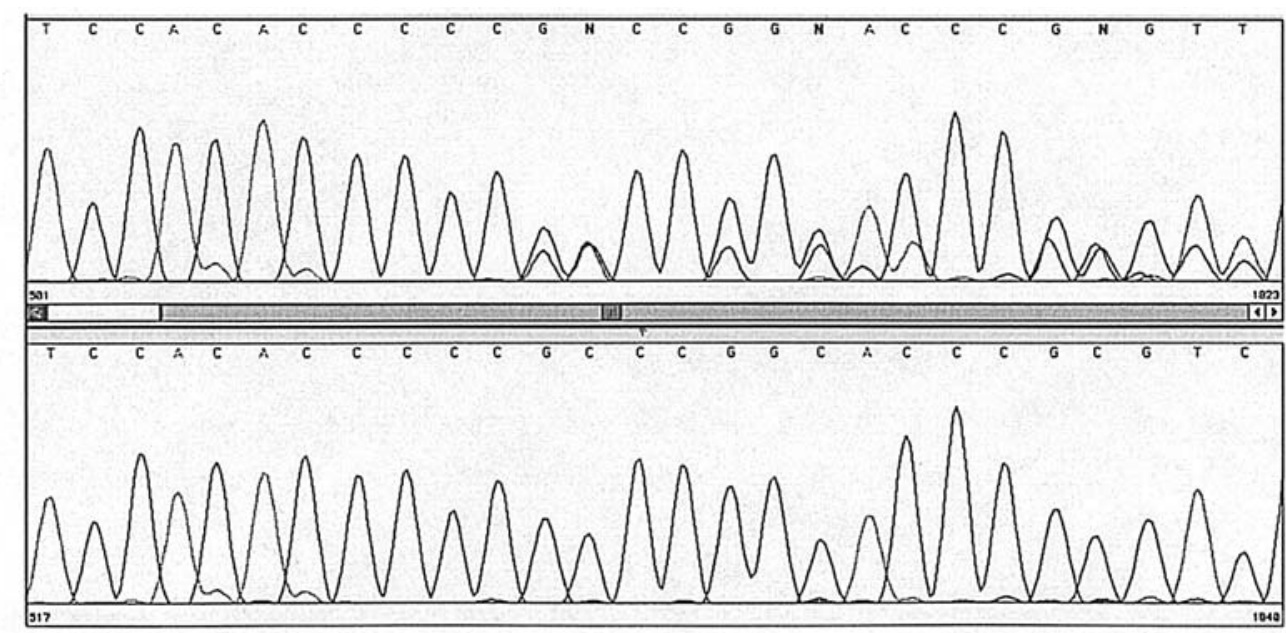

B

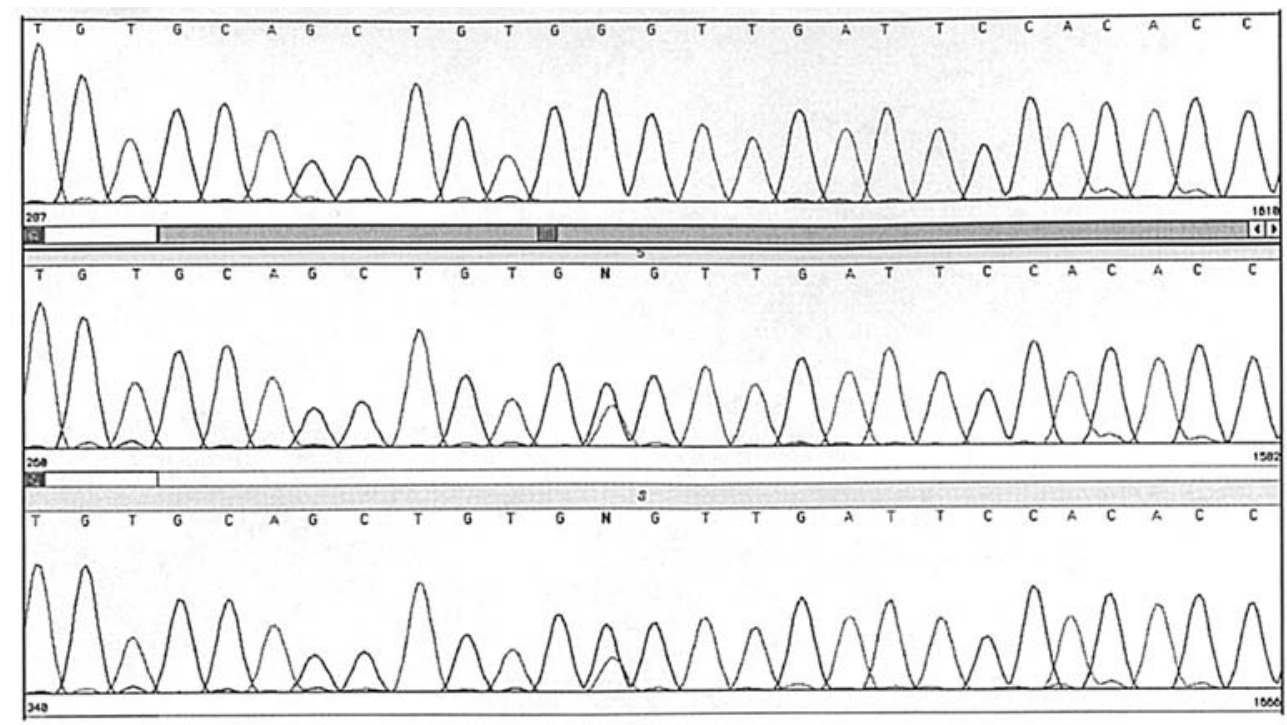

C

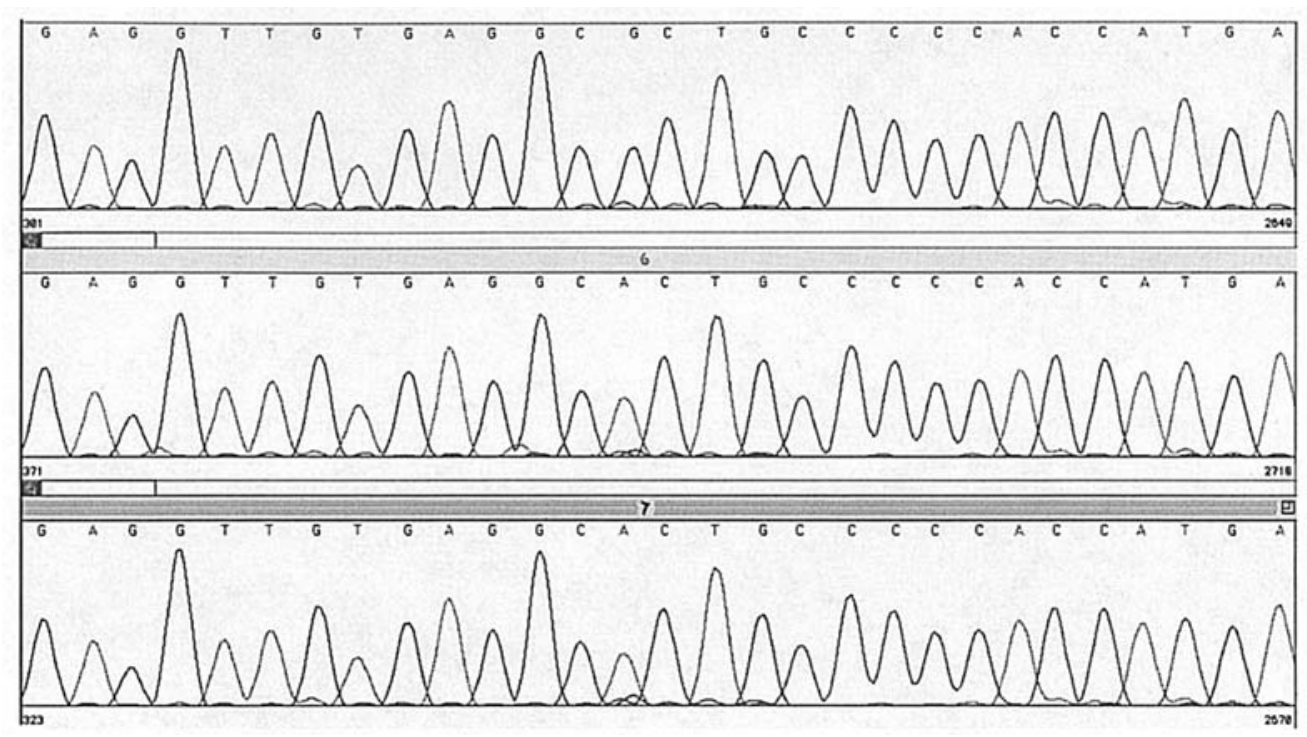

Figure 1. Non-polymorphism p53 mutations found, with their sequencing traces (wild-type given with each figure for comparison). A, Frameshift: exon 5/nucleotide 13129-13130/insertion C. Found in 1 patient (3\%). B, Trp >STOP: TGG>TGA, codon 146, exon 5. Found in 2 patients (6\%). C, Arg $>$ His: CGC>CAC, codon 175, exon 5. Found in 2 patients $(6 \%)$.

arsenic, asbestos, betel quid, coal, cyclophosphamide, heavy metals, mustard gas, pesticides, petrochemicals, phenacetin, chemotherapeutic agents, solvents, radon, radiation, thorotrast, vinyl chloride, psolaren and UV radiation (7).

\section{Results}

Mutations found. Of 31 cholangiocarcinoma DNA samples studied, 24 had evidence of p53 gene alteration, compared to 
the control wild-type DNA. Together, these 24 samples had 48 gene alterations, of which there were 12 different types, as summarised in Table II. However, the five most commonly occurring of these 12 gene modifications were known p53 polymorphisms. Four changes were found in introns. Two of these intron mutations have not previously been described in cholangiocarcinoma (intron 3, nucleotide 11992, C>A; and intron 7, nucleotide 13964, G>C). Of the eight mutations occurring in exons, three were previously documented polymorphisms, of which two were silent and one was a missense mutation (arginine to proline at codon 72 of exon 4). This left five p53 mutations, which were not known polymorphisms, and were not silent. Three would lead to frameshifts (exon 4, nucleotide 12243 , codon 107 , deletion T; exon 5 , nucleotide 13129_13130, codon 151, insertion C; and exon 6, nucleotide 13332_13333, codon 191, deletion CT). The remaining two mutations were $\mathrm{G}>\mathrm{A}$ transitions. One was a nonsense mutation [codon 146 of exon 5 (tryptophan to STOP); and the second was a missense mutation at a CpG site (codon 175 of exon 5: arginine to histidine)]. Examples of the sequence traces obtained for these mutations are shown in Fig. 1. In all but four cases (two intron 2 polymorphisms and two exon 4 polymorphisms), mutations were heterozygous.

To summarise, out of 31 cholangiocarcinoma patient DNA samples, there were five p53 mutations that were not silent or known polymorphisms. This mutation rate of $16 \%$ is comparable to other European studies $(8,10,11)$. The mutations did not relate to any particular ethnic origin in our patient population. The missense mutation at a CpG site (codon 175) found in this study has also been previously reported in studies from Europe and Asia (8,10-12). In contrast, the nonsense mutation has only been previously reported in Europe (8). The three frameshift mutations, in exons 4, 5 and 6 , do not appear to have been previously reported in human cholangiocarcinoma. Two of these frameshifts were due to deletions, and deletions have been previously reported in cholangiocarcinoma from 2 studies. The third was due to an insertion in an exon. Such insertions have not previously been described in human cholangiocarcinoma. Overall, there was no predominant mutational spectrum amongst the set of cholangiocarcinoma tumours samples studied, or on combining these mutations with the dataset of known cholangiocarcinoma p53 mutations (7).

Comparison with known p53 mutations induced by chemical carcinogens. The commonest type of p53 mutation induced by chemical carcinogens according to the IARC database is a $\mathrm{G}: \mathrm{C}>\mathrm{A}: \mathrm{T}$ ( or T:A) transition (7). Often these are induced at $\mathrm{CpG}$ sites, for example by arsenic, asbestos, betel quid, phenacetin, chemotherapeutic agents, radiation, radiotherapy, vinyl chloride and UV radiation. Although such mutations are also the predominant type (up to $30 \%$ of all p53 mutations) found in cholangiocarcinoma, the codon distribution of these mutations is variable. Table III summarises and compares the predominant p53 mutation pattern and affected codons found in cholangiocarcinoma, with p53 changes induced by individual chemical carcinogens, based on information contained in the IARC p53 database (7). As well as in cholangiocarcinoma, G:C>A:T transitions are also specifically the commonest mutation pattern induced by aromatic amines, betel quid, cyclophosphamide, mustard gas, psoralen and UVA, radiation and solvents. However, the codon distribution of these mutations in cholangiocarcinoma and the codon distributions of mutations induced by these chemical carcinogens, did not match. In cholangiocarcinoma, the p53 codons most commonly containing mutations (that is, containing at least $5 \%$ of the total number of single base substitutions) are $175,245,248,273$ and 282. None of the chemical carcinogens reviewed exhibited the same codon distribution with respect to p53 mutations, although several of these codons, amongst others, were targeted by certain chemicals. In particular, betel quid (codons 175, 248, 273), coal $(175,245,273)$ and vinyl chloride $(245,248,273)$ targeted three out of the five codons commonly mutated in cholangiocarcinoma. Overall however, a comparison of the p53 mutations found in this study, as well as of all known p53 mutations, with those reported mutations arising from various carcinogens, did not reveal a consistent pattern or distribution of mutations which could be defined as a mutational fingerprint for cholangiocarcinoma.

\section{Discussion}

There are only five published studies of p53 sequencing in cholangiocarcinoma from Western countries, including three from Europe $(8,10,11,13,14)$. To date, studies have been mostly been done on small groups (patient numbers ranged from 7 to 51 ), and have focused on exons 5-8 (only two studies have sequenced exon 9). No study has investigated mutations along all exons of p53, potentially missing mutations that could be important in terms of a functional role and/or a mutational signature. As a result, up to $80 \%$ of reported missense mutations are in exons 5-8, which code for the sequence-specific DNA binding domain. However, there are several reasons why a search for a mutational signature should also involve the remaining exons, as they also contain important functional signals. The $\mathrm{N}$-terminus of the $\mathrm{p} 53$ protein, important in the transcriptional function of $\mathrm{p} 53$, is encoded by exons $2-4$. The C-terminus, involved in oligomerisation and nuclear localisation of p53, as well as in the recognition of DNA repair and induction of apoptosis (15), is encoded by exons 9-11. Insertions and deletions, which have been seen in human cholangiocarcinoma, but which are less common than single point mutations would be more efficient mutagenic mechanisms than point mutations in disrupting these $\mathrm{N}$ - and C-terminal functional domains. In human cancer studies, when compared to missense mutations, such insertions and deletions in p53 occur more frequently in exons 2-4 and 9-11 than exons 5-8 $(15,16)$. Exon 9 of p53 has only been sequenced in two studies, and exons 2-4 and 10-11 have not previously been investigated in human cholangiocarcinoma, despite the fact that several mutations have been reported in each of these exons at several other tumour sites (7). Furthermore, even silent mutations, or those of no functional consequence, including such mutations outside exons 5-8, may reflect the fingerprint of genetic insult caused by a carcinogenic agent(s) (17). Mutations in introns may also be functionally relevant. For example, an important promoter is located in the 10,738 base-pair first intron at the 5'-end of the p53 gene (16).

This study is the first to examine the whole p53 gene (cDNA) for full mutational spectra in the context of cholangio- 
Table III. Comparison of p53 mutations in cholangiocarcinoma with p53 mutations induced by chemical carcino-

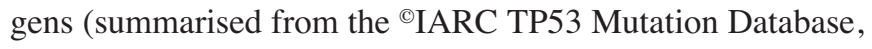
R7 Version).

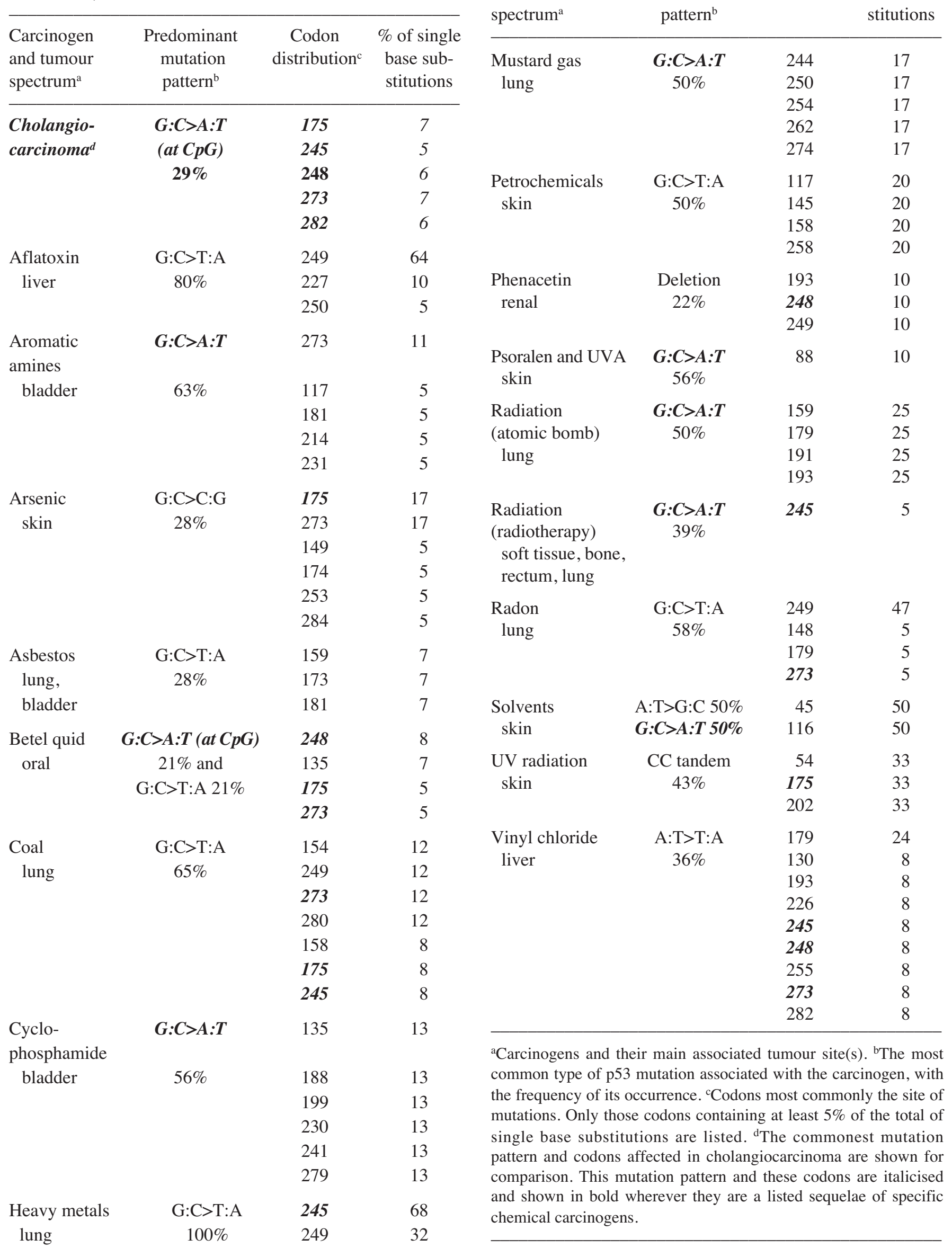

Table III. Continued.

\section{C}

Carcinogen Pred


carcinoma. No specific characteristic pattern of mutations that could be ascribed to a mutational signature was found in the majority of patients. However, two deletions and one insertion were found in 31 patients, none of which have been previously described. These three mutations would all lead to premature termination at the mRNA level. They could not be correlated with any specific aspect of the relevant patients' available medical histories. It is therefore not possible to say how these types of mutation, which are uncommon in cholangiocarcinoma, arose, or whether they are related to carcinogen exposure.

Absence of a mutational signature. There are several possible reasons for lack of mutational signature so far in cholangiocarcinoma. First, this may reflect differences in aetiology in study populations, reflecting geographic, environmental, cultural and socio-economic differences. Larger studies from different regions and cholangiocarcinoma cluster areas are therefore required. Second, a mutational spectrum can potentially involve p53 exons outside 5-8, or even introns; or possibly even a multi-gene mutational fingerprint across several genes. No published studies to date have analysed the fulllength cDNA. Genetic abnormalities other than p53 have been demonstrated in cholangiocarcinoma. Several studies have also found mutations in multiple genes within the same cholangiocarcinoma patients $(18,19)$. Kang and colleagues examined 40 cases and found $15 \%$ had an abnormality in at least two genes from p53, APC, K-ras (18). These findings suggest that carcinogenesis in cholangiocarcinoma may be a multi-step process, involving the activation of various oncogenes and inactivation of tumour suppressor genes. Hence, several different carcinogens may be involved. Therefore, cholangiocarcinoma may depend on an accumulation of mutations of critical regulatory genes in a multi-stage manner, similar to colonic carcinoma. Thus, p53 mutation may be a relatively late genetic event in the carcinogenic cascade, and not the primary target of the carcinogen. Clonal selection and expansion of certain mutations in p53, and/or other genes, along the line of various mutations may occur because they confer an oncogenic potential or growth advantage. Therefore, further investigations on cholangiocarcinoma at developmental stages of the disease, for example, biliary dysplasia, tumour invasion and metastasis are needed. Furthermore, p53 mutations can arise endogenously, in addition to arising from exogenous mutagenic agents, for example from errors in DNA replication, RNA splicing and DNA repair (15).

Mutations in non-coding regions. The search for mutations as the cause of human genetic disease is mainly confined to the promoter region, coding sequences and intron-exon boundaries of the gene under scrutiny. The four commonest gene alterations found in this study were in introns. Two of these were known polymorphisms and the remaining two have not previously been described in cholangiocarcinoma. Two further known polymorphisms were found in exons, one being a missense mutation and the other a silent mutation. There is increasing evidence, although not yet involving p53 or cholangiocarcinoma, that mutations in non-coding regions, and even silent mutations, can be important from a gene function point of view. Examples of this phenomenon include mutations at branchpoints that affect mRNA splicing. Even translationally silent mutations can, theoretically, inactivate genes by influencing splicing accuracy or efficiency (20).

Branchpoint mutations have been associated with human disease, for example congenital contractual arachnodactyly and extrapyramidal movement disorder with branchpoint mutations occurring in genes for lecithin:cholesterol acyltransferase, low density lipoprotein-receptor and the fibrillin-2gene (21-24).

In addition to three previously undescribed frameshift mutations, this study also found two novel intron mutations in cholangiocarcinoma, in introns 3 and 7. The latter mutation in particular ( $\mathrm{G}>\mathrm{C}$ at nucleotide 13964), could possibly be a branchpoint disruptor as it is at a potential branch site region. Branch site mutations affecting mRNA splicing have not yet been reported in p53 and their exact location is unclear. This is probably because they have not previously been looked for specifically. This is a potential problem in all p53 mutational analysis, not just with respect to cholangiocarcinoma. Early studies of p53 mutations were based on sequencing cloned full-length cDNA. Since these initial reports showed that mutations seem to cluster around exons 5-8, subsequent studies have limited their analysis to these exons. Potential mutations in exons 2-4 and 9-11 are underrepresented, as are introns. This is an important source of data bias, as the type of p53 mutation is known to differ between central and peripheral coding regions, with a higher proportion of nonsense mutations, deletions and insertions occurring in peripheral regions.

Sources of bias affect the description of p53 mutations in databases. Focused p53 analysis on selected areas of the genome is one of several causes of bias that must be acknowledged when interpreting p53 mutations and comparing them with specialist databases such as the IARC database used in this study. Other causes of bias and error which affect p53 mutation reporting include small study sizes, mistakes in reporting codon and base sequences, the use of retrospective samples with a lack of corresponding epidemiological data, and differences in DNA retrieval and pre-screening methods (25). Immunohistochemistry is often used to select cholangiocarcinoma tissues for further mutational analysis by sequencing, as many missense mutations induce stabilisation of p53 leading to its intracellular accumulation. This is another source of bias however, as positive immunohistochemistry does not always correlate with p53 mutational status. In particular, mutations resulting in a $\mathrm{p} 53$ protein-null phenotype (nonsense mutations, insertions and deletions) represent up to $20 \%$ of all p53 mutations (25).

Other factors that need to be considered in the search for human cancer gene fingerprints include the high genetic instability of cancer cells that may increase the frequency of mutations in certain cancer genes regardless of exposure factors, and the importance of DNA repair mechanisms and of the corresponding degree of population variation (26). It is also important to consider the possibility of epigenetic alterations of p53 expression, for example by methylation of its promoter region. Such a mechanism has been proposed to link the development of oesophageal squamous cell carcinoma to exposure to chemical carcinogens such as 
nitrosamines (27). Furthermore, DNA damage may be multifactorial and possibly complicated by random events, for example DNA adduct formation and damage secondary to chronic inflammation, oxidative stress etc. Finally, given the above possibilities, there simply may not be a specific carcinogenic agent and therefore no specific mutational signature.

Future studies. Several improvements can be made in the way future p53 mutational investigations are carried out to increase the possibility of detecting a mutational signature and thus isolating a causative agent for cholangiocarcinoma. Larger studies, from different regions and cluster areas, are required as cholangiocarcinoma has a relatively low frequency of p53 mutations and larger numbers of samples need to be analysed to find mutations. It is also relevant to analyse nontumour DNA to confidently exclude a germ line origin for any alterations. Studies should clearly identify histological type, site (intra- or extrahepatic), grade and stage of all samples, as well as documenting sex, age, ethnicity and individual risk factors, such as smoking, diet, medical and drug history, infection, urban or rural dwelling, and occupational history. The majority of patients providing cholangiocarcinoma tissue for this investigation had died prior to the start of the study, and not all the aforementioned information was available from clinical notes. Future prospective data sets should ideally include this information.

In future studies, full mutational spectra, including noncoding regions and intron-exon boundaries, should be sought. Complete descriptions of all mutations found should be given, including codon, nucleotide, exact base change and consequence (amino acid substitution, insertion, deletion etc). Some mutations described in published studies may result from laboratory errors such as contamination and polymerase errors. These can be minimised by encouraging repeat analysis of all positive tumours to confirm the presence of mutation, ideally in a second, independent laboratory. This would be particularly important with respect to rare and unusual mutations, but is unlikely to happen without scientific journals encouraging this practice with their editorial policies. The IARC database claim a policy of contacting authors of reports containing unclear data for clarification, but the response rate is poor (25). Finally, the effects of point mutations, including intronic mutations, should ideally be analysed at the mRNA level before drawing conclusions about the importance of the affected amino acid, as their correct classification in terms of the actual mechanism of gene inactivation is essential for understanding structural and functional interrelationships in the corresponding protein $(20,25)$. Hence future studies should investigate whether identified p53 mutations disrupt p53 function, or lead to its aberrant expression. Ideally, p53 mutations should be analysed at different stages of cholangiocarcinogenesis to ascertain their role along the carcinogenic pathway. However, given the relatively low occurrence of cholangiocarcinoma, its typically late clinical presentation and the absence of well-defined precursor lesions, such studies would be difficult to carry out.

Although this study reports three previously unknown mutations of p53 in cholangiocarcinoma, we were unable to reveal a characteristic mutational signature. Nevertheless, it is worthwhile pursuing the further molecular characterization of cholangiocarcinoma, a fatal, increasing disease with a very poor prognosis and a lack of effective treaments. Increasing our understanding of the relationship between structure and function of mutations and the corresponding proteins, is potentially important not just for enlightening on aetiology, but also because this may lead to earlier diagnosis, better prognostic markers and more effective therapies.

\section{Acknowledgements}

We are grateful to Nigel Gooderham (Department of Biological Chemistry, Imperial College London) and David CuthbertHeavens, Claire Taylor and Tanja Crnogorac-Jurcevic (Cancer Research UK) for helpful discussions and assistance with the laboratory work. We are also very grateful to Cancer Research UK, Medical Research Council, for supporting this work.

\section{References}

1. Khan SA, Taylor-Robinson SD, Toledano MB, Beck A, Elliott P and Thomas HC: Changing international trends in mortality rates for liver, biliary and pancreatic tumours 1979-1997. J Hepatol 37: 806-813, 2002.

2. Pfeifer GP: p53 mutational spectra and the role of methylated CpG sequences. Mutat Res 450: 155-166, 2000

3. Hollstein M, Hergenhahn M, Yang Q, Bartsch H, Wang ZQ and Hainaut P: New approaches to understanding p53 gene tumor mutation spectra. Mutat Res 431: 199-209, 1999.

4. Smela ME, Currier SS, Bailey EA and Essigmann JM: The chemistry and biology of aflatoxin $\mathrm{B}(1)$ : from mutational spectrometry to carcinogenesis. Carcinogenesis 22: 535-545, 2001.

5. Bergquist A, Glaumann H, Stal P, Wang GS and Broome U: Biliary dysplasia, cell proliferation and nuclear DNA-fragmentation in primary sclerosing cholangitis with and without cholangiocarcinoma. J Intern Med 249: 69-75, 2001.

6. Arora DS, Ramsdale J, Lodge JP and Wyatt JI: p53 but not bcl-2 is expressed by most cholangiocarcinomas: a study of 28 cases. Histopathology 34: 497-501, 1999.

7. IARC, International Agency for Research on Cancer, p53 Database. 2002. (www.iarc.fr/p53).

8. Tullo A, D'Erchia AM, Honda K, Kelly MD, Habib NA, Saccone C and Sbisa E: New p53 mutations in hilar cholangiocarcinoma. Eur J Clin Invest 30: 798-803, 2000.

9. Gupta RC: Non-random binding of the carcinogen N-hydroxy2-acetylaminofluorene to repetitive sequences of rat liver DNA in vivo. Proc Natl Acad Sci USA 81: 6943-6947, 1984.

10. Torre G, Pasquini G, Pilotti S, et al: TP53 mutations and mdm2 protein overexpression in cholangiocarcinomas. Diagn Mol Pathol 9: 41-46, 2000

11. Jonas S, Springmeier G, Tauber R, et al: p53 mutagenesis in Klatskin tumors. Hum Pathol 29: 955-960, 1998.

12. Kiba T, Tsuda H, Pairojkul C, Inoue $S$, Sugimura $T$ and Hirohashi S: Mutations of the p53 tumor suppressor gene and the ras gene family in intrahepatic cholangiocellular carcinomas in Japan and Thailand. Mol Carcinog 8: 312-318, 1993.

13. Sturm PD, Baas IO, Clement MJ, et al: Alterations of the p53 tumor-suppressor gene and $\mathrm{K}$-ras oncogene in perihilar cholangiocarcinomas from a high-incidence area. Int $\mathrm{J}$ Cancer 78: 695-698, 1998.

14. Tannapfel A, Sommerer F, Benicke M, et al: Genetic and epigenetic alterations of the INK4a-ARF pathway in cholangiocarcinoma. J Pathol 197: 624-631, 2002.

15. Harris CC: Structure and function of the p53 tumor suppressor gene. J Natl Cancer Inst 88: 1442-1445, 1996.

16. OMIM ${ }^{\mathrm{TM}}$ (Online Mendelian Inheritance in Man) Database. Ed. McKusick VA. National Centre for Biotechnology Information. 2002. (http://www.ncbi.nlm.nih.gov/Omim).

17. Kamikawa T, Amenomori M, Itoh T, et al: Analysis of genetic changes in intrahepatic cholangiocarcinoma induced by thorotrast. Radiat Res 152: S118-S124, 1999. 
18. Kang YK, Kim WH, Lee HW, Lee HK and Kim YI: Mutation of p53 and K-ras, and loss of heterozygosity of APC in intrahepatic cholangiocarcinoma. Lab Invest 79: 477-483, 1999.

19. Cong WM, Bakker A, Swalsky PA, et al: Multiple genetic alterations involved in the tumorigenesis of human cholangiocarcinoma: a molecular genetic and clinicopathological study. J Cancer Res Clin Oncol 127: 187-192, 2001.

20. Cartegni L, Chew SL and Krainer AR: Listening to silence and understanding nonsense: exonic mutations that affect splicing. Nat Rev Genet 3: 285-298, 2002.

21. Kuivenhoven JA, Weibusch H, Pritchard PH, Funke H, Benne R, Assmann G and Kastelein JJ: An intronic mutation in a lariat branchpoint sequence is a direct cause of an inherited human disorder (fish-eye disease). J Clin Invest 98: 358-364, 1996.

22. Webb JC, Patel DD, Shoulders CC, Knight BL and Soutar AK: Genetic variation at a splicing branch point in intron 9 of the low density lipoprotein (LDL)-receptor gene: a rare mutation that disrupts mRNA splicing in a patient with familial hypercholesterolaemia and a common polymorphism. Hum Mol Genet 5: 1325-1331, 1996 .
23. Maslen C, Babcock D, Raghunath $\mathrm{M}$ and Steinmann B: A rare branch-point mutation is associated with missplicing of fibrillin-2 in a large family with congenital contractural arachnodactyly. Am J Hum Genet 60: 1389-1398, 1997.

24. Janssen RJ, Wevers RA, Haussler M, et al: A branch site mutation leading to aberrant splicing of the human tyrosine hydroxylase gene in a child with a severe extrapyramidal movement disorder. Ann Hum Genet 64: 375-382, 2000

25. Hernandez-Boussard T, Montesano R and Hainaut P: Sources of bias in the detection and reporting of p53 mutations in human cancer: analysis of the IARC p53 mutation database. Genet Anal 14: 229-233, 1999.

26. Vineis P: Molecular epidemiology of cancer. In: The Cancer Handbook. Alison MR (ed.) Nature Publishing Group, pp377-387, 2002.

27. Zhang L, Lu W, Miao X, Xing D, Tan W and Lin D: Inactivation of DNA repair gene O6-methylguanine-DNA methyltransferase by promoter hypermethylation and its relation to p53 mutations in esophageal squamous cell carcinoma. Carcinogenesis 24: 1039-1044, 2003. 